\title{
ELEMENTOS PARA LA ELABORACIÓN DE PLANES DE TUTORÍA EN LA UNIVERSIDAD
}

\author{
Joaquín Gairín, \\ Mònica Feixas, \\ Jordi Franch, \\ Cristina Guillamón \\ y Dolors Quinquer* \\ Universidad Autónoma de Barcelona
}

RESUMEN: La tutoría, entendida como conjunto de actuaciones dirigidas a apoyar el pleno desarrollo personal y académico del estudiante universitario, se considera, cada vez más, como un indicador de calidad. Bien organizada, contribuye a mejorar la adaptación y rendimiento de los estudiantes, a la vez que mejora la imagen pública de la universidad.

Bajo una perspectiva actual, la tutoría se considera un valor añadido para cualquier titulación, al incluir acciones muy diversas: académicas, administrativas, centradas en el conocimiento de uno mismo y en los aprendizajes, abarcadoras del tiempo de permanencia en la institución universitaria, etc. No obstante, su éxito depende en gran parte del grado de articulación e institucionalización que se consiga a través de un plan de acción global (Plan de Acción Tutorial).

Toda planificación debe partir del análisis de necesidades, concretar un plan de actuación (que, además de explicitar objetivos y recursos, delimita el modelo tutorial y la distribución /coordinación de funciones) y establecer mecanismos de seguimiento y mejora. El conocimiento, por último, de algunos errores que cabe evitar, posibilita la creación de propuestas técnicamente justificadas y defendibles.

ABSTRACT: Tutorship, understood as a series of actions aimed at supporting the full personal and academic development of University students is increasingly considered as a quality indicator. If it is properly organized, it helps to improve the adjustment and performance of the students and also boosts the public image of the institution involved.

\footnotetext{
* Miembros del Grupo de Orientación y Tutoría de la Universidad Autónoma de Barcelona, coordinados por Joaquín Gairín y financiado por el DURSI, convocatoria de 2002.
} 
Nowadays, tutorship is considered as an added value in any diploma as it includes very varied actions: academic and administrative issues, actions focused on the knowledge of the self and learning processes, plans covering the whole stay at University, etc. However, its success depends to a great extent on the level of coordination and institutionalisation reached through a global action plan (Tutorship Action Plan).

Any planning must start with an analysis of the needs to devise an action plan (which, apart from explaining its objectives and resources, must specify the tutorship model chosen and the distribution/coordination of roles) and determine monitoring and improvement mechanisms. Finally, the knowledge of some mistakes to be avoided allows to create technically justified and defensible proposals.

PALABRAS CLAVE: Tutoría, enseñanza superior, Plan de Acción Tutorial.

KEYWORDS: Tutorship, higher education, Tutorship action plan.

\section{Introducción}

Gran parte del alumnado universitario presenta importantes carencias en materia de orientación académica, profesional y personal, tanto antes de acceder a la universidad, durante sus estudios, como también antes de la entrada al mundo del trabajo. Hasta el momento, las respuestas ofrecidas por la universidad han sido parciales y desvinculadas entre sí, aunque también debemos reconocer que existen experiencias exitosas en algunas universidades como puedan ser los programas de estudiantes asesores, los de orientación para la inserción laboral, las tutorías personalizadas o el acompañamiento durante la transición de la secundaria a la universidad.

Lo que todas las universidades han evidenciado es que los alumnos requieren de orientación y ésta debe ser diferenciada según el momento en que se encuentran con relación a sus estudios universitarios. Dar respuesta a estas necesidades pasa por pensar qué papel juega la orientación dentro de la política de enseñanza y aprendizaje de la universidad y cómo se concreta en planes de tutorías en cada facultad.

Mejorar la tutoría se sitúa así en el marco de la mejora de la docencia universitaria y está en el contexto de las acciones que las universidades toman dirigidas a aumentar la calidad de los servicios que otorgan y su prestigio institucional. Se entiende así como un servicio para el estudiante, al mismo tiempo que útil para la universidad.

La importancia de la tutoría para la universidad nunca ha sido puesta en duda y ha sido explícitamente reconocida en informes sobre la universidad (Bricall, 2000), recomendada por organismos universitarios (la Agencia para la Calidad del Sistema Educativo en Cataluña ya la recomendaba en su informe de 1999) y señalada en otros contextos (así, los pilares propuestos por el Informe Delors de 1996 incluían una visión tutorial). Las ventajas que se le asignan tienen que ver con:

- Su identificación como un indicador de calidad. Las funciones tutoriales de información, formación y orientación son valiosas para los estudiantes e influyen en la prevención de los retrasos y abandonos de itinerarios profesionales.

- La diversidad de la oferta formativa y la complejidad de algunos planes de estudio hacen necesario un proceso de orientación hacia el estudiante. 
- La identificación de necesidades y delimitación de puntos fuertes y débiles, útiles para generar planes de mejora.

Para los estudiantes, la tutoría se sitúa en el marco de una formación completa que no sólo les considera desde el punto de vista instructivo. Permite, además:

- Ayudar a ajustar diferencias ante la diversidad de procedencias y niveles de los estudiantes ante los procesos formativos.

- Mejorar la satisfacción del estudiante al proporcionar respuestas a demandas y necesidades de los mismos.

- Contribuir a la adaptación a la universidad y a disminuir las posibles dificultades vinculadas al proceso de capacitación.

No es extraño, por tanto, que la tutoría se considere un valor añadido para cualquier titulación, nexo de conexión entre los estudiantes y la universidad, facilitadora de las transiciones entre secundaria-universidad y mundo laboral, explicitación de los procesos de orientación deseables en cualquier proceso formativo y un instrumento que ayuda a mejorar la imagen pública de la universidad.

Su necesidad se hace cada vez más evidente. Sin embargo, no deberíamos olvidar que los enfoques recientes incluyen bajo su denominación un campo de trabajo amplio que incluyen acciones muy diversas: académicas, administrativas, centradas en el conocimiento de uno mismo y en los aprendizajes, dirigidas a todo el tiempo de permanencia en la universidad, etc.

Atender las diferentes opciones exige utilizar enfoques globales, lejos de las prácticas y posibilidades de muchos centros universitarios donde los dispositivos tutoriales están poco desarrollados y cuando lo están no todos los estudiantes hacen de los mismos la utilización que sería deseable.

Apuntamos en los próximos apartados un conjunto de elementos, deudores en gran parte de la aportación de Quinquer y Sala (2002), que pueden servir para definir y concretar los planes de acción tutorial que las universidades quieran desarrollar.

\section{¿Qué entendemos por Plan de Acción Tutorial?}

El Plan de Acción Tutorial (PAT) es un documento de gestión institucional a medio-largo plazo que explicita la organización de las tutorías de un centro o de una titulación. Como plan que articula un conjunto de acciones y procesos de apoyo y asesoramiento para los estudiantes, tiene que ser flexible y debe incluir decisiones tanto en la dimensión académica del estudiante como en la profesional y la personal. Concretamente podría contemplar (Hernández de la Torre, 1998; Quinquer y Sala, 2002):

- la adaptación a la universidad,

- la elección de asignaturas y configuración del propio currículo,

- el fomento del autoconocimiento,

- pensar sobre sí mismo y sobre los demás (autocontrol),

- proporcionar medios por abordar situaciones de la vida real,

- la mejora del rendimiento académico,

- desarrollar técnicas que clarifiquen los valores, 
- desarrollar habilidades cognitivas y destrezas de estudio,

- la orientación académica respeto a las dificultades en la materia y la orientación profesional: individual y social,

- facilitar información sobre el medio, e

- informar sobre la demanda laboral, la transición a la vida activa y los itinerarios laborales.

La planificación de la acción tutorial es fundamental en cualquiera acto educativo y necesaria durante el proceso de aprendizaje del alumno. Toda planificación de esta naturaleza debe considerar al menos cuatro fases: la primera plantea el diseño de finalidades, objetivos y recursos; la segunda el tiempo y las actividades (distribución de funciones y coordinación y cooperación); la tercera concreta la realización de actividades en las que intervienen el análisis, los métodos e instrumentos, y la cuarta fase pretende la valoración del funcionamiento de las tutorías como organización y de los resultados concretos del proceso orientador. Todo ello puede concretarse de varias maneras optando en nuestro caso por la propuesta que se presenta en el cuadro 1 y que desarrollamos en próximos apartados.

\begin{tabular}{|l|}
\hline La detección de necesidades: \\
\hline - las necesidades formativas y de orientación de los estudiantes \\
\hline - las necesidades de los estudios \\
\hline Las finalidades y los objetivos: \\
\hline - generales (con las finalidades de orientación) \\
\hline - específicos (por ámbitos) \\
\hline Los contenidos: \\
\hline - el alcance del plan (a qué estudiantes se dirige) \\
\hline - las modalidades priorizadas \\
\hline Los momentos clave: \\
\hline - transición secundaria-universidad \\
\hline - durante los estudios universitarios \\
\hline - transición universidad-mundo laboral \\
\hline La tipología de tutorías: \\
\hline - según la naturaleza \\
\hline - según la actividad \\
\hline Los implicados: \\
\hline - alumnos (alumnos-asesores) \\
\hline - tutores \\
\hline - coordinadores \\
\hline - otros profesores y profesionales \\
\hline El seguimiento del Plan de tutoría \\
\hline La evaluación del Plan de tutoría \\
\hline
\end{tabular}

Cuadro 1: Principales elementos de un Plan de Acción Tutorial (Quinquer y Sala, 2002) 


\section{La detección de las necesidades de los estudiantes y de los estudios}

La elaboración de un PAT implica que los responsables académicos o el profesorado que lo elabore tenga en consideración y analice principalmente:

- las necesidades informativas, formativas y de orientación de sus estudiantes,

- las características del centro y las necesidades específicas de los estudios dónde se aplicará, y

- las prácticas de atención a los estudiantes ya consolidadas (la sesión informativa sobre el plan de estudios, las tutorías de asignatura, las tutorías de practicums...).

Las encuestas y entrevistas (a individuos y a grupos) tanto de estudiantes como de docentes pueden ser los instrumentos más idóneos por identificar las necesidades, los problemas y los aspectos mejorables, a la vez que facilitan la participación. También el análisis de datos sobre los estudios (características de los estudiantes de nuevo ingreso, número de matriculados por cursos, número de deserciones, porcentaje de asignaturas aprobadas en la primera convocatoria...).

La recogida de toda esta información tiene que permitir (Quinquer y Sala, 2002):

- Conocer el perfil del estudiante universitario (porcentaje según el género, edad, motivaciones y vías seguidas por escoger estudios), la procedencia académica (bachillerato, formación profesional, mayores de 25 años, CUECE, otras titulaciones, etc.), expectativas sobre los estudios y las salidas profesionales.

- Informarse de sí se han cursado determinadas asignaturas que se consideran importantes para la titulación.

- Identificar la preparación básica que según los docentes se requiere para cursar los estudios (conocimientos y competencias básicas que se podrían considerar importantes para la titulación).

- Conocer los datos de matrícula y las acciones de apoyo al alumnado que ya se realizan o se han realizado en los últimos cursos.

- Analizar los resultados académicos, el número de abandonos y sus causas, las bolsas de estudiantes que no se presentan o suspenden reiteradamente determinadas materias, el tiempo real de duración de los estudios, etc.

- Valorar las acciones de atención al alumnado que ya se realizan, por ejemplo, las acciones de acogida, si hay un buen aprovechamiento de las tutorías de asignatura $u$ otras acciones.

Un ejemplo de estudio sobre necesidades de orientación de estudiantes universitarios es el realizado por Ausín y otros (1997) en la Facultad de Educación de la Universidad de Salamanca. Se centran en tres aspectos: la orientación académica, la orientación profesional y la orientación personal:

- La orientación académica hace referencia a información sobre como funciona la universidad, la facultad, el contenido de la carrera, otras carreras, la iniciación a la investigación, becas, cursos, seminarios, información sobre los derechos de los alumnos, asesoramiento en la formalización de la matrícula, asesoramiento en la elección de asignaturas optativas y de libre elección, en casos de vulneración de los derechos, injusticias..., organización de conferencias, seminarios, cursos... y y desarrollo de técnicas de estudio.

- La orientación profesional contempla ofrecer información sobre salidas profe- 
sionales, orientación laboral (que hacer al acabar la carrera, como hacer un currículum vitae...), organización de actividades para conectar al alumno con el mundo laboral, y programas de desarrollo de habilidades para la profesión.

- Finalmente, la orientación personal hace referencia al apoyo para la resolución de problemas personales, información sobre actividades extraacadémicas, información sobre voluntariado, asociacionismo juvenil, ONG... y orientación en valores morales.

Del estudio se desprende que principalmente los servicios de orientación académica, profesional y personal recaen sobre todo en el Servicio de Orientación de la universidad, aunque hay otros agentes que también colaboran (profesores, delegación de alumnos y otros profesionales). Asimismo se manifiesta la necesidad de impulsar servicios de orientación enfocados y planificados según unos planteamientos modernos y tomando elementos positivos de otras experiencias universitarias, así como crear servicios de orientación a nivel de facultad. Estos no tendrían que descartar la participación de paraprofesionales -en especial la orientación a través de otros estudiantes o peer counseling-, más que un sistema tutorial llevado a cabo por profesores. "El alumno no percibe al profesor universitario como principal agente de la orientación, aunque sí considere importante su tarea en algunas actividades concretas" (Ausín y otros, 1997:49).

\section{Finalidades, objetivos, contenidos y agentes}

La concreción de los objetivos está vinculada al análisis de las necesidades detectadas y sirve para establecer las prioridades y concretar los ámbitos de actuación. La información recopilada tiene que permitir identificar aquellos aspectos dónde es más necesaria la acción tutorial, ya sea facilitar la transición y el acogimiento de los estudiantes a la universidad, apaciguar determinados déficits que se han detectado, orientarles en la organización de su currículum, la orientación profesional u otras cuestiones.

Como ejemplos de objetivos de la acción tutorial sirven los redactados por Hernández de la Torre y Domínguez (1998):

- Responder a las necesidades individuales de los alumnos universitarios, conociendo y valorando al alumno individualmente.

- Personalizar la educación universitaria, orientando de manera individual al alumno.

- Orientar los procesos de aprendizaje universitario para ayudar en la toma de decisiones.

- Formar al estudiante universitario técnica y científicamente poniendo al día los conocimientos existentes.

- Ayudar en la búsqueda de soluciones de problemas individuales.

- Potenciar el aprendizaje.

La organización de un Plan de Tutoría implica tomar decisiones sobre contenidos diversos, especificando el alcance que tiene que tener: se ha de establecer si sólo se dirigirá a los estudiantes de una asignatura, estudiantes de primer curso, si se hará extensivo a todo el primer ciclo, a los de prácticum, a los que tienen dificultades o a todos los que cursan la titulación. En esta decisión influyen la priorización de necesidades, la disponibilidad de tutores, los recursos disponibles, etc. 
Por ello, los contenidos pueden variar y podrían ser, entre otros:

1) Acciones de información y de orientación

2) Formación para la mejora de los métodos de aprendizaje

3) Tutoría de asignatura

4) Asesoramiento para temas concretos y problemas diversos

5) Atención en situaciones especiales

6) Orientación para el prácticum

7) Orientación para la inserción laboral

Las funciones tutoriales pueden, asimismo, ser ejercidas por agentes diversos:

- Profesores de diversas asignaturas.

- Tutores/as seleccionados especialmente para hacer estas funciones entre el profesorado.

- Estudiantes de cursos superiores que informan y orientan a otros (tutoría entre iguales).

- Profesionales en el lugar de trabajo (los tutores de prácticum) que actúan de manera coordinada con el tutor/a de la universidad.

- Personal de programes específicos (tutores de programas de intercambio).

En síntesis, vemos que las modalidades de acción tutorial son múltiples. En cada caso, el Plan debe decidir como articula los contenidos a desarrollar con los posibles agentes y destinatarios.

\begin{tabular}{|c|c|c|}
\hline $\begin{array}{l}\text { Contenido de las acciones } \\
\text { tutoriales }\end{array}$ & Destinatarios & Agentes \\
\hline $\begin{array}{l}\text { - Información sobre la } \\
\text { institución y orientación } \\
\text { en la elección de itinerarios, } \\
\text { de asignaturas... } \\
\text { - Formación para la mejora } \\
\text { de los métodos de aprendizaje } \\
\text { - Apoyo académico de asignatura } \\
\text { - Asesoramiento sobre temas } \\
\text { concretos y problemas diversos } \\
\text { - Atención en situaciones } \\
\text { especiales } \\
\text { - Orientación para el } \\
\text { prácticum } \\
\text { - Orientación para la } \\
\text { inserción laboral }\end{array}$ & $\begin{array}{l}\text { - El estudiante } \\
\text { individualmente } \\
\\
\text { - Los estudiantes } \\
\text { en grupo }\end{array}$ & $\begin{array}{l}\text { - Un profesor /a } \\
\text { - Otros estudiantes } \\
\text { (tutoría entre iguales) } \\
\text { - Un programa } \\
\text { específico } \\
\text { - Un profesional } \\
\text { del lugar de } \\
\text { prácticas }\end{array}$ \\
\hline
\end{tabular}

Cuadro 1. Contenidos, agentes y destinatarios del Plan de Acción Tutorial (Quinquer y Sala, 2002:26) 


\section{Tipología de tutorías}

Podríamos distinguir dos tipologías diferentes de tutorías, en función de si se estudia la naturaleza de la misma (personalizada, grupal, virtual, autoorientación, en parejas...) o el tipo de actividad (transición secundaria-universidad, académica, transición universidad-mundo laboral, de prácticum, de programas de intercambio...).

\section{A. TIPOLOGÍAS EN FUNCIÓN DE SU NATURALEZA:}

La mayoría de contenidos se pueden vehicular mediante tutorías individualizadas o grupales, aunque pueden utilizarse otras vías únicas o complementarias como la tutoría virtual, la tutoría por parejas, la tutoría de asignatura o la autoorientación.

La tutoría personalizada consiste en el apoyo, basado en la relación personal y directa, que recibe el estudiante por parte de un tutor/a. El contenido de este apoyo puede ser diverso aún cuando el eje de la acción tutorial personalizada tendría que conjugar la vertiente académica con el asesoramiento más personal, por ejemplo:

- el análisis conjunto con el tutor/a de la trayectoria del estudiante con el fin de recibir orientación para resolver dificultades académicas o tomar decisiones sobre la propia carrera,

- consejo en relación con problemas con los que se pueda encontrar en la vida universitaria, ya sea porque compagina estudio y trabajo o estudio-trabajo-familia, o bien en relación con problemas económicos, de salud u otros. A menudo se trata de canalizar las demandas de los estudiantes hacia servicios y profesionales especializados de la misma universidad.

En todo caso, esta vía personalizada debería ir más allá de ofrecer respuestas puramente informativas que se podrían vehicular mejor por otros canales (webs, carteles informativos, información personalizada, etc). Señalar, asimismo, que algunos planes de tutorías establecen tutorías personalizadas en las cuales se asigna a cada alumno un tutor para toda la duración de estudios.

En el supuesto de que la tutoría sea grupal, los grupos pueden formarse mediante la asignación de un determinado número de alumnos a un profesor-tutor o a través de la matriculación en una asignatura específica de tutoría.

Las ventajas de la tutoría grupal pueden concretarse en:

- Es un marco muy interesante para tratar temas comunes, compartir cuestiones del proceso académico, averiguar necesidades y problemas o para intercambiar experiencias y especialmente para recibir ayuda, puesto que el grupo actúa como elemento de apoyo para la orientación y la formación. En este sentido, la interacción profesor-alumnos se complementa con la interacción entre iguales.

- El tutor/a puede recoger información valiosa y contrastada desde la perspectiva de los estudiantes de interés para una mejor organización y gestión de los estudios. También permite recopilar información sobre las necesidades y características de los estudiantes, sobre aspectos de la docencia y para detectar y canalizar carencias diversas como, por ejemplo, cuestiones de infraestructura, de funcionamiento de determinados servicios, disfunciones en la coordinación, etc.

Se aconseja, al respeto, lo siguiente:

- Para garantizar la asistencia hace falta establecer algún mecanismo de recompensa para los estudiantes que participan en estas acciones tutoriales, como, 
por ejemplo, la obtención de créditos en el marco de las asignaturas optativas o de libre elección.

- El contenido de las sesiones tiene que ser contextualizado y útil para los estudiantes como, por ejemplo, para orientar la transición a la universidad y conocer el contexto universitario, para planificar el propio trabajo y hacerlo más productivo, para conocer recursos y estrategias de trabajo propias de la titulación, para orientarse en las posibles especializaciones y construirse el itinerario curricular, para el aprovechamiento del prácticum, para la transición al mundo laboral, para conocer las salidas profesionales, etc.

La tutoría virtual, que en su día se asociaba al dominio de la educación a distancia, acontece en la actualidad como un recurso para muchos profesores y estudiantes. Las tutorías electrónicas se presentan hoy como entornos complementarios en los procesos de comunicación entre estudiantes y profesores, cada uno con sus propias características que lo hacen apto para una situación en que no es posible el contacto real. Mediante la tutoría virtual el profesor puede ofrecer propuestas de ejercicios y orientaciones para realizarlos, proponer una tarea, evaluar los resultados y sugerir propuestas de mejora, de recuperación o ejercitación adicional.

Bien organizada por el profesor, la tutoría electrónica se convierte en un revulsivo que cambia el sentido del trabajo de los alumnos. Este es un aspecto importante que algunos detractores de la tecnología olvidan: es cierto que la tecnología por sí sola no mejora la enseñanza, pero también es cierto que se ha mostrado capaz de mejorarla actuando como generadora de innovación y estimuladora del cambio metodológico.

La incorporación de las nuevas tecnologías ha posibilitado en muchas universidades una amalgama de actividades complementarias a las propiamente presenciales, que bien planificadas (materiales, estrategias didácticas, evaluación) ofrecen un entorno a aprendizaje más dinámico, motivador y real. Pero si no se estructuran de manera adecuada, provocan en el estudiante un sentimiento de aislamiento y sobrecarga de contenido. La concepción pedagógica del entorno y las estrategias de aprendizaje que se utilizan serán en gran parte decisivas en el éxito de la tutoría.

No obstante y por el momento, la tutoría virtual es más aceptada en estudios semipresenciales o a distancia que en estudios plenamente presenciales. En estos últimos, el profesor realiza tutorías frente a frente y las complementa en muchos casos con la tutoría virtual. Si no se hace un uso más extendido de la tutoría electrónica es por algunos de los problemas o dificultades que comporta la nueva proximidad en la relación alumno-profesor y la pérdida de espacio y tiempo.

Los estudiantes que se comunican de manera virtual con los profesores a menudo quieren una respuesta inmediata (comunicación, información, documentación) que muchas veces los profesores no pueden dar. Esta situación se podría solucionar, por ejemplo, si se difundiera claramente que se destina el mismo horario de tutorías presenciales a responder los mensajes de las tutorías virtuales.

Aún así, queda mucho campo para explorar con relación a la efectividad de la tutoría presencial y virtual y, concretamente, con relación al contenido (dirigidas o libres) o modalidad (individuales, grupales) más adecuada.

La tutoría en parejas es una modalidad donde estudiantes experimentados (normalmente formados previamente y con una compensación de créditos de libre elec- 
ción, ayudas monetarias, becas u otras opciones para la carrera) proporcionan apoyo (informativo, de acompañamiento u otros) a nuevos estudiantes.

La tutoría de asignatura es un aspecto más de la función docente que se podría potenciar en muchas titulaciones. El profesorado destina como parte de su dedicación unas horas de tutoría semanales, fuera del horario de clase, para atender y orientar más personalmente a los estudiantes en cuestiones relacionadas con las materias que imparte.

El éxito académico está influido por la estructuración adecuada de las tareas y actividades de aprendizaje, que son el eje central de la vida del aula. Estas tareas son además responsables de las demandas, de los procesos de aprendizaje y del comportamiento de los alumnos. Cuando los criterios de éxito no están bien definidos se da ambigüedad en el intercambio académico e incertidumbre en el alumnado. Una buena orientación curricular comporta, al respecto, seguridad, autoestima y éxito y se hace necesaria durante todo el proceso de enseñanza y aprendizaje de la materia.

Finalmente, sobre la autoorientación en la universidad señalar que ha sido una práctica común hasta la aparición de servicios de orientación y de apoyo al estudiante. Durante mucho tiempo, el estudiante ha tenido que buscar información y tomar decisiones curriculares y profesionales sobre la base de criterios personales más o menos acertados. La creación de gabinetes psicopedagógicos y de orientación y el establecimiento de acciones tutoriales ha contribuido a mejorar la fundamentación de las decisiones tomadas.

Existe poca bibliografía sobre la autoorientación ejercida por el mismo estudiante en lo referido a instrumentos, estrategias o prácticas. En la actualidad, los materiales para la autoorientación se suelen centrar en la mejora de hábitos de estudio y técnicas de aprendizaje, en cuestionarios sobre intereses profesionales o itinerarios curriculares, en estrategias para la búsqueda de trabajo (presentación de currículums, entrevistas), organización del tiempo u otros similares.

\section{B. TIPOLOGIAS EN FUNCIÓN DE LA ACTIVIDAD}

Hay determinados momentos claves en la carrera universitaria que son especialmente importantes para que los estudiantes se representen qué es la universidad, definan su proyecto de vida y tomen decisiones para hacer los ajustes adecuados. Disponer de apoyo y de asesoramiento puede ser especialmente útil y orientador. Estos momentos son:

1. La transición desde las diversas vías de acceso a la universidad (la secundaria, la formación profesional, mayores de 25 años o demás) cuando los recién llegados necesitan acogida, información y orientación.

2. Durante el curso de los estudios les hace falta orientación académica, especialmente en aquellos momentos en que se tienen que tomar decisiones de futuro, optar entre determinadas especialidades, aún cuando también se puede necesitar ayuda para apaciguar déficits de aprendizaje o, incluso, en una dimensión más personal y vital.

3. La transición al mundo del trabajo, cuando los estudiantes tienen que hacer prácticas, especialmente fuera de la universidad, y cuando, en los últimos cursos, les es útil disponer de información sobre los perfiles profesionales, las salidas laborales u otras cuestiones que les puedan ayudar a orientar la carrera 
profesional como puedan ser la elaboración de currículums o el desarrollo de habilidades sociales y de comunicación.

\section{B.1. La Acogida a los estudiantes de primero:}

Durante la transición desde la secundaria o desde otras vías de acceso al sistema universitario se produce un cambio cualitativo en las demandas que se hacen a los estudiantes, un cambio del contrato implícito entre el estudiante y la institución académica, que estos se tienen que representar adecuadamente para circular sin sustos.

Cuándo los estudiantes han de iniciar los estudios universitarios se sienten contentos y angustiados a la vez: contentos porque inician una nueva etapa de su vida, una nueva aventura donde aprenderán junto a nuevos compañeros, donde probablemente se sentirán más libres y tendrán que organizarse de manera más autónoma; angustiados porque no saben si afrontarán lo suficientemente bien las nuevas demandas y porque el cambio de "contrato" suele conllevar una cierta incertidumbre ante las propias motivaciones y capacidades. De ahí que a lo largo del primero curso sea útil una tutorización específica en forma de acogida y de asesoramiento que les ayude a orientarse y los guíe en la transición a los estudios universitarios.

Con el nombre de "acogida" se denomina al conjunto de acciones orientadas a facilitar a los nuevos estudiantes el conocimiento del contexto -edificio, compañeros, profesorado, plan de estudios, funcionamiento del centro, servicios con que cuenta, etc.- y también de las demandas de la institución universitaria de tal modo que se hagan una representación inicial.

Su objetivo principal es crearles una predisposición positiva hacia el aprendizaje y también conseguir que se sientan adecuadamente atendidos por la institución, fidelizarles y contribuir a una buena imagen pública de la universidad. Por todo ello, los procesos de acogida deberían dirigirse a todo el colectivo de los estudiantes de primero, no solamente a unos cuántos voluntarios.

Dadas las finalidades que se persiguen con los planes de acogida, es especialmente importante la manera como se llevan a cabo las acciones programadas, que siempre requieren un trato afectuoso hacia los estudiantes, una mirada positiva que les anime a los estudios y les confirme su acierto en la elección de aquella titulación y universidad.

Ejemplos de posibles acciones a desarrollar para la acogida de los estudiantes podrían ser las recogidas por Quinquer y Sala (2002:36-42) :

- Acciones iniciales de acogida, como juegos, visitas, etc., dirigidas a romper el aislamiento individual de los estudiantes y a facilitar contactos entre las personas de un mismo grupo.

- Sesiones informativas en grupos reducidos, programadas con el proceso de matrícula y en la que intervienen profesores específicos e incluso alumnos experimentados.

- Tutoría personalizada para primer curso, que permite ofrecer al estudiante una persona de referencia ante sus problemáticas personales y académicas.

- Tutoría entre iguales (peer tutoring), ya comentada.

- Asignaturas introductorias, dirigidas a mejorar el conocimiento de las opciones de estudios, la base académica, la mejora de técnicas de estudio u otras opciones que ayuden a los estudiantes a optimizar su esfuerzo. 
- Sesiones técnicas de estudio, dirigidas a profundizar en aspectos concretos como puedan ser las estrategias de trabajo de la carrera (realización de mapas, localización de textos...) o técnicas de estudio (tomar apuntes, estrategias de lectura rápida, gestión del tiempo personal, organización de la agenda...).

- Guías de asignatura, como programa desarrollado que recoge aspectos habituales (objetivos, temáticas, metodología y evaluación) junto a otros como: descripción detallada del sentido de la asignatura, requisitos previos, desarrollo previsto de las clases, libros básicos comentados, estructura de los trabajos demandados, ejemplo de pruebas de evaluación, etc.

Muchas de estas acciones vienen acompañadas de actuaciones previas que tratan de facilitar una transición adecuada. Algunas acciones posibles serían:

- Jornadas de puertas abiertas

- Información previa a la matriculación

- Cursos propedéuticos o introductorios

- Visitas comentadas de dependencias

- Sesiones de acogida programadas para los primeros días

- Sesiones abierta sobre temas científicos

- Charlas informativas

La conexión con los centros de secundaria se estima cada vez más importante. Así, la Universidad Autónoma de Barcelona ha puesto en funcionamiento el Programa Argó que trata de acompañar a los estudiantes en el difícil viaje de la secundaria a la universidad. Algunas de las actividades que ofrece son: asesoramiento a trabajos de investigación de enseñanza media, estancia de estudiantes en la universidad, premios a trabajos de investigación, banco de recursos para estudiantes y profesores de secundaria y soporte y orientación en el proceso de transición.

\section{B.2. El seguimiento de los estudiantes:}

El seguimiento de los estudiantes puede hacerse a partir de las acciones de los profesores que actúan como tutores o con la existencia de tutores específicos. Algunas acciones habituales en este momento de los estudios son:

- Orientación específica en el momento de seleccionar especialidad, materias optativas o itinerarios.

- Sesiones de trabajo de coordinación entre el profesorado: jornadas de docencia, jornadas monográficas...

- Sesiones de coordinación entre profesores de varias asignaturas.

- Sesiones de valoración entre el profesorado y representantes de estudiantes.

- Tutorías individuales o en pequeño grupo, obligatorias o no, para el seguimiento del trabajo de algunas asignaturas.

- Desarrollo de la docencia tutorizada y de la tutoría integrada dentro de las asignaturas.

- Creación de foros telemáticos y otras actividades de tutoría virtual (e-mail, trabajos en red...) 
- Programas especiales para estudiantes con necesidades educativas especiales, extranjeros, personas con fracaso en los estudios, etc.

- Salidas externas y colectivas relacionadas con la carrera.

- Orientación a servicios especiales (unidades de asesoramiento psicopedagógico, por ejemplo).

El seguimiento de los estudiantes justifica en todo este proceso la importancia de la tutoría personalizada y de las entrevistas que forman parte de los procesos de ejecución.

\section{B.3. Orientación para la transición al mundo del trabajo:}

La transición de los estudiantes al mundo del trabajo se está convirtiendo en una cuestión de interés creciente para la universidad. La posibilidad real de employability de sus graduados es una cuestión clave, con una incidencia nada despreciable en la captación de nuevos estudiantes y en la valoración de determinados estudios.

Además, la apertura de la universidad al mundo laboral tiene que ser forzosamente un elemento importante para la transformación de los estudios que se ofrecen, y tiene que contribuir muy especialmente a transformar las formas más tradicionales de enseñar, que tendrán que adaptarse a una realidad social y profesional en cambio constante. En este contexto debe considerarse como un valor añadido el que la universidad conecte con el mundo profesional y se preocupe de ayudar a sus estudiantes en el proceso de inserción mediante acciones tutoriales diversificadas.

Según la Agencia para la Calidad del Sistema Universitario a Catalunya, las acciones universitarias para la inserción sociolaboral de los estudiantes se puedan clasificar en cuatro categorías:

- Prácticum o asignatura que tiene como objetivo la aplicación práctica de los conocimientos adquiridos en centros de trabajo y que consta con este nombre en el Plan de Estudios.

- Prácticas relacionadas con las asignaturas: prácticas que tanto puedan formar parte de una asignatura o ser una asignatura independiente, pero que en ambos casos tienen como objetivo la aplicación práctica de asignaturas de carácter teórico.

- Prácticas profesionalizadoras o prácticas en empresas: prácticas que no constan en el plan de estudios y se realizan en centros de trabajo.

- Proyecto Fin de Carrera.

Más allá de la existencia de prácticas o de prácticums, las acciones de inserción profesional incluyen otras acciones como: sesiones informativas (conferencias, exposiciones, jornadas, presentación de empresas...), documentación adecuada, sesiones con exgraduados, etc.

La atención a alumnos de prácticas tiene así que gobernarse por principios de:

- profesionalización: capaz de otorgar un valor añadido a la formación práctica

- globalidad: abordarse desde una metodología capaz de facilitar a los estudiantes una percepción global y aplicada del ejercicio de la profesión

- transversalidad: materia transversal de todas las diferentes disciplinas académicas 
- supervisión: permite al estudiante integrar conocimiento teórico y práctico y construir esquemas de actuación profesional

- integración/colaboración: tiene que garantizar siempre la integración de la docencia en el mundo de la práctica

- indagación: que capacite al alumno para formular y comprobar hipótesis sobre su manera de actuar

- multifuncionalidad/multicontextualidad: que permita encarar la formación práctica desde un modelo de practicum orientado a la flexibilidad y a la capacidad de adaptación del estudiante a múltiples funciones y contextos de actuación.

La orientación para la transición tiene por objetivo, básicamente, el proporcionar información sobre las salidas profesionales, las estrategias para buscar trabajo, las técnicas para el autoempleo, las habilidades sociales (trabajo en equipo, creatividad, resolución de problemas, asertividad), etc. La tutoría en esta dirección debería ayudar a abrir perspectivas, promover procesos de autoevaluación, ayudar a planificar el proceso de inserción, fomentar la creación de planes de carrera, comprometer a los profesionales que empiezan con determinados valores, ayudarles a ubicarse profesionalmente (asociaciones, colegios...), etc.

\section{B.4. La atención a colectivos específicos:}

Las universidades suelen contar con un programa de integración o apoyo al estudiante con discapacidad con el objetivo de promover la satisfacción de las necesidades educativas especiales así como su integración en el entorno universitario. A través de un servicio de acogida, atención y asesoramiento, se estructura un programa global de actuaciones para garantizar las soluciones más adecuadas para estos estudiantes durante el tiempo de estancia a la universidad, hasta el acceso al mundo profesional.

Los estudiantes extranjeros de los programas de intercambio Erasmus y Tempus (universidades europeas) o de otros programas de movilidad (universidades no europeas) presentan una serie de necesidades académicas (de asesoramiento y evaluación de los diferentes currículos universitarios, y las derivadas del desarrollo de las actividades académicas y prácticas docentes) y sociológicas (con relación a la competencia lingüística, la integración y adaptación a la vida universitaria, la relación con estudiantes locales y alojamiento) que hay que atender.

El apoyo específico que reciben consiste en:

- antes de llegar a la universidad de destino, información sobre la universidad, los planes de estudio y las potenciales asignaturas a realizar,

- una vez en la universidad, atención personalizada de la respectiva Oficina de Relaciones Internacionales,

- atención individualizada por parte de los coordinadores de titulación y de intercambio. Estos actúan como tutores de estos alumnos a la hora de establecer la tabla de equivalencias entre las asignaturas que se ofrecen en la universidad de origen y las que finalmente se realizan en la universidad de destino, contribuyen también a resolver dudas o dificultades en aspectos académicos. 


\section{Los implicados}

Además de los estudiantes, los principales implicados en el proceso de planificación, desarrollo y evaluación de un Plan son los tutores (de asignatura, de curso, de programas específicos...) y aquellos colaboradores que también intervienen en el proceso de orientación (estudiantes asesores...).

El tutor es aquel encargado de orientar y dar apoyo a los alumnos de un curso o asignatura y conseguir de ellos que desarrollen sus potencialidades al máximo. Es "aquel profesor que tiene la responsabilidad de supervisar el desarrollo cognitivo, personal y profesional de un número determinado de estudiantes, los cuales pueden o tienen que tomarlo como referente" (Gallego, 1997:68). Según este autor, el tutor debería poseer un:

- Perfil personal (SER): autoaceptación como persona y como profesional, estabilidad emocional, seguridad en sí mismo, aceptación de las limitaciones, respeto al valor y la dignidad de las personas, empatía hacia los estudiantes.

- Perfil cognitivo (SABER): conocer el proyecto de la universidad y estar de acuerdo con sus presupuestos, identificarse con la historia de la institución y con las ideas fundamentales, las políticas generales y con las específicas referidas a la tutoritzación de alumnos; conocer las relaciones entre el contexto y los factores socioculturales y la influencia en el desarrollo humano; y elaborar programaciones de la acción tutorial.

- Perfil técnico (HACER): incluye conocer técnicas a utilizar en la acción tutorial: habilidades de comunicación efectiva, capacidad de confrontar opiniones, capacidad de pensamiento reflexivo, desarrollar programaciones hechas, mantener contactos con el servicio de orientación, con otros compañeros, estrategias de supervisión.

- Perfil afectivo (ESTAR): un buen autoconocimiento, saber expresar las propias necesidades, saber identificar las propias expectativas, ser tolerante con la propia frustración y estrés, respetar la individualidad de cada estudiante y conocer los criterios éticos y códigos deontológicos.

- Perfil social (SABER ESTAR): presentar conductas de calma y coraje ante circunstancias difíciles, saber reír en el momento apropiado, tomar el estado de humor más apropiado para cada circunstancia.

En otras palabras, el tutor universitario tendría que ser un profesor con un buen conocimiento de los estudios, con interés por orientar y a yudar los estudiantes y con competencias por hacerlo. Básicamente tendría que cumplir requisitos como:

- Conocimiento amplio de la titulación, de los itinerarios curriculares, de los ámbitos profesionales, de los servicios que ofrece el campus, con acceso a la información, también experiencia de participación activa en la vida de la institución, etc.

- Habilidades sociales y de relación: aptitudes para la relación interpersonal y la comunicación, empatía con los estudiantes, actitud por abordar los problemas de una manera positiva, etc.

La ayuda que puede prestar a los estudiantes se puede concretar en aspectos de carácter:

- Académico: facilitando información y acogimiento, siguiendo el rendimiento 
del estudiante, orientándolo en la elección de asignaturas, en la manera de estudiar y en las técnicas de trabajo intelectual, etc.

- Profesional: en la orientación de su itinerario profesional, en la formación continuada.

- Personal: orientándolo en relación a los especialistas o los servicios que puedan ayudarlo en situaciones problemáticas, ayudándolo en la toma de decisiones o en la resolución de conflictos, etc.

Respecto a la coordinación de tutores, ésta debería centralizar la captación, la formación, el seguimiento y la valoración del trabajo hecho por los tutores y tutoras; también, proporcionar recursos y propuestas de acción.

En lo que se refiere a la formación, puede prepararse al tutor con una:

- formación inicial de información y de estudio de las características de la facultad o escuela donde desarrollará su tarea. Tendrá que concienciarse de la importancia de la tarea encomendada y asimilar unas bases teóricas sobre la acción tutorial. Este periodo puede organizarse como un seminario de unas 5 horas de duración aproximadamente.

- formación continuada. Gallego (1997) propone al respecto:

- reuniones periódicas de intercambio de experiencias entre tutores,

- reuniones con temáticas propuestas por el servicio de orientación,

- dossieres informativos con información sobre la universidad y/o facultad, junto con otras lecturas que se consideren útiles por el desarrollo de la acción tutorial, y

- creación de un centro de documentación especializado, dónde se recojan materiales publicados con referencia a la acción tutorial en educación superior.

A esto se le pueden añadir cursos o talleres para conocer el sistema de tutorías implantado, la organización de los estudios, seminarios para compartir vivencias, informaciones, reflexiones y documentación con objeto de analizar conjuntamente las acciones que se realizan.

El papel del coordinador de primero también merece una atención específica. El primer curso académico en la vida de un estudiante universitario es decisivo para el éxito o fracaso de su permanencia en los estudios y disponer de un profesor de referencia durante este proceso se considera beneficioso. En algunas universidades, se ha creado la figura de coordinador de primer curso al cual Quinquer y Sala (2002: 3334) le asignan funciones como:

- Coordinar acciones de acogida de los estudiantes y velar por una transición fácil del alumnado a los estudios universitarios.

- Centralizar y coordinar las acciones tutoriales que se programen, ya sean de información, de orientación o de apoyo.

- Identificar las características principales del alumnado de primero: los estudios a través de los que acceden a la universidad, los conocimientos sobre programas informáticos y la disponibilidad de uso que tienen, las lenguas extranjeras que conocen y el nivel logrado, las tareas o los trabajos que desarrollan además de estudiar, las materias que cursan, las salidas profesionales a las que desearían optar u otra información que se considere de interés para el profesorado a la hora de impartir las clases. 
- Velar por una buena distribución de materias, de horarios, de servicios, de las horas de tutoría, etc. realizadas en beneficio de los estudiantes.

- Coordinar al profesorado que imparte clase a primero, con objeto de integrar los esfuerzos individuales, dinamizar de una manera conjunta los recursos disponibles e introducir la cultura de la coordinación entre el profesorado.

- Analizar y valorar los resultados obtenidos de los estudiantes con datos cuantitativos: número de no presentados, de suspensos, de aprobados, etc. Por ejemplo, analizando el éxito y el fracaso por asignatura y entregando los resultados comparados al profesorado.

- Analizar y valorar las percepciones de los estudiantes sobre el primer curso a la universidad desde una perspectiva cualitativa, con entrevistas o encuestas.

- Evaluar el trabajo realizado en la coordinación con la delimitación de los puntos fuertes y de los puntos débiles, propuestas de mejora, etc.

Los programas de estudiantes-asesores se caracterizan por el asesoramiento que estudiantes de cursos superiores, con experiencia en la carrera, ofrecen a compañeros de cursos inferiores en aspectos académicos como hábitos o técnicas de estudio y en aspectos curriculares como itinerarios, asignaturas, cargas lectivas. Se trata de una experiencia que está cobrando cada vez más adeptos entre las universidades. La idea que subyace a dicha experiencia es la de garantizar que los estudiantes de primer curso tomen sus decisiones académicas importantes con la información suficiente, antes de que sea demasiado tarde y que cuenten con las herramientas psicopedagógicas necesarias por abordar con éxito sus objetivos académicos.

\section{El seguimiento y evaluación de la acción tutorial}

El diseño del Plan, la puesta en práctica y los resultados obtenidos tienen que ser objeto de seguimiento y de evaluación a fin de verificar hasta qué punto el proceso es adecuado y se logran los objetivos previstos, también como mecanismo de mejora y optimización.

Sesiones de evaluación de los estudiantes, reuniones de seguimiento del Plan, sesiones de revisión de prácticums, reuniones de coordinación de titulaciones, etc. son momentos idóneos para la realización de algunas actuaciones (recogida de datos, análisis de resultados, elaboración de conclusiones, recomendaciones, etc.) que permiten hablar de la tutoría como una acción que se puede ir siguiendo a lo largo del curso.

El seguimiento empieza ya en la elaboración del Plan con la consiguiente asignación de tutores y tutoras y dotación de contenido y estrategias a la acción tutorial. La propuesta del Plan debería analizarse desde su adecuación al contexto, su capacidad para satisfacer necesidades, su viabilidad y evaluabilidad; también considerando la coherencia interna del diseño y el grado de concreción del producto deseado y del proceso para su realización.

Organizar bien el Plan y su ejecución exige la participación de los implicados desde el primer momento. Parece pues apropiado responsabilizar a los participantes de las acciones del seguimiento y de la evaluación de las actividades realizadas, seleccionando la información y plasmándola en instrumentos de recogida de datos. Además, los responsables del Plan ejercerán funciones de motivación, coordinación y supervisión de las actividades para garantizar el desarrollo del seguimiento. 
Si bien a final de un curso escolar se puede poner en común la efectividad de la acción tutorial, la evaluación de ésta sería casi imposible si no se han dado los pasos necesarios de recogida de información sobre el funcionamiento del Plan. Por ello, insistimos en que será importante determinar qué datos se recogen y de qué manera, además de nombrar a un responsable.

La evaluación a realizar durante el proceso o al final del mismo debería de responder a preguntas como (Quinquer y Sala, 2002:31-32):

a) ¿Por qué evaluar? La evaluación permite rectificar aquellas cuestiones que lo requieren y ajustar con más precisión los diversos elementos que componen el plan. Por ejemplo, en el caso de la tutoría personalizada comprobar si el número de estudiantes asignados a cada tutor/a es el adecuado, verificar la utilidad que para los estudiantes tiene este servicio, comprobar si hacen un uso suficiente, confirmar que se están atendiendo las necesidades específicas y reales de los estudiantes, determinar si la información que reciben los tutores es suficiente o bien si hace falta complementarla, etc.

b) ¿Quién evalúa? La opinión de los estudiantes es muy importante, puesto que son los verdaderos destinatarios y beneficiarios del plan. También interesa el punto de vista de los tutores y tutoras que conocen las incidencias, problemas y las posibles disfunciones. Finalmente, es conveniente contar con la visión institucional que pueden dar los coordinadores del Plan y de la titulación.

c) ¿Qué se evalúa? Haría falta determinar aquellos aspectos o cuestiones con relación a los cuales se quiere verificar la adecuación y el buen funcionamiento. Con la finalidad de determinar las cuestiones que se quieren evaluar se pueden emplear como referentes: los objetivos, las modalidades, las acciones que se realizan, la selección de tutores, el apoyo que reciben los tutores, los recursos invertidos u otras cuestiones, aún cuando posiblemente el aspecto clave sea la utilidad que tienen las acciones tutoriales para los estudiantes.

d) ¿Cómo evaluar? Para recopilar información necesaria, pueden ser útiles los cuestionarios dirigidos a cada uno de los colectivos de los cuales se quiere conocer su percepción. La información se puede complementar y/o contrastar con entrevistas individuales o en grupo (una muestra representativa puede ser suficiente).

Los cuestionarios puede incluir proposiciones cerradas sobre las cuales se pide el grado de acuerdo o desacuerdo; también preguntas abiertas para captar mejor la opinión y obtener una información más cualitativa, más rica en matizaciones y aportaciones personales, aun cuando puede ser más difícil de procesar. Esta modalidad es especialmente adecuada cuando se evalúa la opinión de grupos numerosos.

e) ¿En qué momentos? Si se pretende el ajuste de los recursos y de las estrategias hace falta hacer un seguimiento del proceso, mientras que si se pretende la valoración del plan en su conjunto (el grado de consecución de los objetivos, la participación de los estudiantes, la utilidad para los estudiantes y la institución universitaria o de otras cuestiones) es mejor una evaluación final al acabar el curso académico.

Los resultados de la evaluación de la acción tutorial pueden aportar información para optimizar su organización tanto respecto a la institución como académicamente; por ejemplo: introducir cambios a la asignación de profesor tutor, cambiar la proporción tutor/estudiante, organizar sesiones de formación e intercambio de tutores, detectar necesidades específicas en los alumnos que requieran acciones de orienta- 
ción y formación más generales, etc. (véase al respecto indicaciones en www.ub.es/tutorsub/manual/index.htm)

Con este proceso, el profesor obtiene un feedback objetivo de su actuación como tutor a la vez que constituye un elemento de reflexión y motivación para el cambio e implicación en el Plan. También, con respecto al alumno, supone normalmente una mejora en su situación académica, tanto desde el punto de vista del rendimiento como en su trayectoria académica (mayor racionalización de la matriculación de créditos, mejor conocimiento de la universidad y sus recursos, etc.).

\section{Posibilidades y dificultades en la elaboración de un Plan de Acción Tutorial (PAT)}

El desarrollo en la práctica de cada uno de los elementos de un Plan de Acción Tutorial en la universidad tiene sus ventajas pero también conlleva una serie de dificultades. A continuación analizamos y sintetizamos en el cuadro 2 y a modo de conclusión, los principales problemas que se asocian a la elaboración de un Plan.

\begin{tabular}{|c|c|c|}
\hline $\begin{array}{l}\text { Elementos } \\
\text { de un PAT }\end{array}$ & Principales problemas & Mejorar las posibilidades de éxito \\
\hline Planificación & $\begin{array}{l}\text { Ofrecer un programa que } \\
\text { no satisfaga las necesidades } \\
\text { reales de orientación de los } \\
\text { estudiantes universitarios. } \\
\text { Falta de detección de } \\
\text { necesidades } \\
\text { Oferta de acciones inconexas, } \\
\text { falta de coordinación entre los } \\
\text { implicados (profesores de } \\
\text { asignatura, coordinadores de } \\
\text { titulación, servicios de } \\
\text { orientación universitaria, } \\
\text { bolsa de trabajo...) } \\
\text { Poca motivación para } \\
\text { incorporar cambios }\end{array}$ & $\begin{array}{l}\text { Buen plan de detección de necesidades de orienta- } \\
\text { ción. Determinar el origen de la demanda, evaluar } \\
\text { las carencias, priorizar. } \\
\text { Sensibilizar a la comunidad universitaria } \\
\text { Delimitación clara de finalidades, objetivos y con- } \\
\text { tenidos, vinculada al análisis de necesidades. } \\
\text { Consignar las estrategias más coherentes con los } \\
\text { objetivos planteados } \\
\text { Liderazgo claro en el ámbito de facultad del/de los } \\
\text { responsable/s del PAT. Capacidad de dinamiza- } \\
\text { ción, implicación y apoyo a las iniciativas que se } \\
\text { adopten } \\
\text { Coordinar las diferentes acciones e implicados en } \\
\text { el proceso. Definir roles y funciones. Acordar el } \\
\text { proceso y los mecanismos de participación y con- } \\
\text { trol } \\
\text { Identificar el potencial comunicativo de los posi- } \\
\text { bles participantes, estudiar el grado de integración } \\
\text { en las relaciones personales e institucionales. } \\
\text { Contar al principio con profesorado motivado } \\
\text { Valorar los costes, riesgos y oportunidades de } \\
\text { innovar }\end{array}$ \\
\hline Desarrollo & $\begin{array}{l}\text { No contar con un plan de } \\
\text { actuaciones concreto: acciones } \\
\text { paso a paso, ni una planificación } \\
\text { logística para llevarlas a cabo } \\
\text { Movilización de recursos } \\
\text { desafortunada }\end{array}$ & $\begin{array}{l}\text { Vehicular la tipología de tutorías más adecuada en } \\
\text { cada caso y en función de cada momento (transi- } \\
\text { ción a la universidad, transición al mundo del tra- } \\
\text { bajo...): } \\
\text { - Ofrecer un programa de tutoría personalizada a } \\
\text { los alumnos de primer y últimos cursos }\end{array}$ \\
\hline
\end{tabular}




\begin{tabular}{|c|c|c|}
\hline $\begin{array}{l}\text { Elementos } \\
\text { de un PAT }\end{array}$ & Principales problemas & Mejorar las posibilidades de éxito \\
\hline & & $\begin{array}{l}\text { Vehicular la tipología de tutorías más adecuada en } \\
\text { cada caso y en función de cada momento (transición } \\
\text { a la universidad, transición al mundo del trabajo...): } \\
\text { - Ofrecer un programa de tutoría personalizada a los } \\
\text { alumnos de primer y últimos cursos } \\
\text { - Verificar las dificultades y posibilidades de la tutoría } \\
\text { virtual y el grado y tipo de respuesta a las demandas } \\
\text { de los alumnos } \\
\text { - Organizar tutorías grupales para contenidos especí- } \\
\text { ficos (informaciones, intercambio de experiencias, } \\
\text { estrategias de estudio...) } \\
\text { - Promocionar la tutoría en pareja y la orientación a } \\
\text { través de estudiantes asesores } \\
\text { - Colectar y elaborar materiales para la auto-orienta- } \\
\text { ción, centrados en aspectos universitarios generales } \\
\text { (hábitos de estudio...) y aspectos concretos (propios } \\
\text { de cada titulación o facultad) } \\
\text { Coordinarse con todos los profesionales (bolsa de tra- } \\
\text { bajo, servicio de psicopedagogía y orientación uni- } \\
\text { versitaria, programas para la atención a alumnos con } \\
\text { NEE...) } \\
\text { Realizar informes de eficacia o detección de dificul- } \\
\text { tades, establecer mecanismos de retroalimentación }\end{array}$ \\
\hline $\begin{array}{l}\text { Seguimiento } \\
\text { y evaluación }\end{array}$ & $\begin{array}{l}\text { Poca supervisión de los } \\
\text { diferentes momentos de la } \\
\text { acción tutorial (acogida, durante } \\
\text { los estudios, la transición a la } \\
\text { universidad) y las diferentes } \\
\text { acciones que se programan } \\
\text { (sesión puertas abiertas, } \\
\text { estudiantes-asesores, tutoría de } \\
\text { asignatura, auto-orientación...) }\end{array}$ & $\begin{array}{l}\text { Contar con instrumentos, estrategias y recursos } \\
\text { humanos con tareas bien diferenciadas en el proce- } \\
\text { so de seguimiento y evaluación: } \\
\text { - Confección de un informe evaluativo global en } \\
\text { base al plan de observación y seguimiento y a los } \\
\text { informes que provengan de los procesos de revi- } \\
\text { sión } \\
\text { - Crítica y reflexión sobre el proceso y la práctica. } \\
\text { Detección de disfunciones. Búsqueda de solucio- } \\
\text { nes a los problemas planteados. }\end{array}$ \\
\hline Institucionalización & $\begin{array}{l}\text { Falta de cultura de orientación } \\
\text { universitaria } \\
\text { Dificultades ante la } \\
\text { incorporación } \\
\text { y sostenibilidad de las } \\
\text { innovaciones implementadas }\end{array}$ & $\begin{array}{l}\text { Incorporación de nuevos hábitos y técnicas en el } \\
\text { clima organizativo y relacional } \\
\text { Creación de dinámicas que empujen hacia la adop- } \\
\text { ción de nuevas estructuras y patrones de comporta- } \\
\text { miento } \\
\text { Establecer mecanismos de apoyo. Formación de tuto- } \\
\text { res. Reconocimiento de las tareas tutoriales en forma } \\
\text { de períodos sabáticos, reducción de docencia, com- } \\
\text { plemento económico... } \\
\text { Control sobre la sostenibilidad de las acciones más } \\
\text { allá de las personas que las iniciaron }\end{array}$ \\
\hline
\end{tabular}

Cuadro 2: Identificación de dificultades y posibilidades de éxito en la implantación de un PAT en la universidad 
Seguramente, un buen PAT podría evitar parte de las problemáticas mencionadas. Sin embargo, el conseguirlo es consecuencia de una voluntad política mantenida, una cultura de la tutoría desarrollada y una alta responsabilidad de los implicados. Exige, también la búsqueda y resolución equilibrada de las múltiples posibilidades de acciones que existen (cuadro 3)

\begin{tabular}{|c|c|}
\hline Individual & Grupal \\
\hline Personal & Institucional \\
\hline Esporádica & Continua \\
\hline Personal & Académica \\
\hline Presencial & Virtual \\
\hline Voluntaria & Obligatoria \\
\hline Del estudiante & Del profesor \\
\hline Única & Diferenciada \\
\hline .................... & ........... \\
\hline
\end{tabular}

Cuadro 3: Alternativas en el caso de la tutoría

En este proceso no hemos de olvidar que las opciones más estables y eficaces son las institucionales, siempre y cuando partan de un concepto más amplio de tutorías, busquen la multivariedad de actuaciones (variadas alternativas y programas de apoyo complementarios), contemplen medidas estructurales (incentivos a los tutores, número adecuado de alumnos por tutor) y no olviden centrarse en las necesidades que la enseñanza- aprendizaje exige a los estudiantes.

\section{BIBLIOGRAFÍA}

ÁLVAREZ, V. (2000): Orientación en los procesos de enseñanza-aprendizaje en la universidad. En SALMERÓN, H. y LÓPEZ, V.L.: Orientación educativa en las universidades. Granada: Grupo Editorial Universitario.

APODACA, P.; ARBIZU, F.; LOBATO, C. y OLALDE, C. (Comps.)(1997): Orientación universitaria y evaluación de la calidad. Universidad País Vasco.

AUSíN, T. y otros (1997): Análisis de necesidades de orientación en alumnos universitarios. En APODACA, P.; ARBIZU, F.; LOBATO, C. Y OLALDE, C. (Comps.)(1997): Orientación universitaria y evaluación de la calidad. Universidad País Vasco.

BORONAT, J. (2000): Enfoque comunitario y dimensión personalizada de la orientación universitaria. En SALMERÓN, H. y LÓPEZ, V.L. : Orientación educativa en las universidades. Granada: Grupo Editorial Universitario.

BRICALL, J. y otros (2002): Informe Universidad 2000. Madrid: CRUE.

CEPES (1994): Counselling and orientation of students in higher education, v. XIX, 3.

DEL RINCÓN, B. (2000): Tutorías personalizadas en la universidad. Ediciones de la Universidad de Castilla-La Mancha. 
DELORS, J. y otros (1996): La educación encierra un tesoro. Informe a la UNESCO de la Comisión Internacional sobre la educación para el siglo XXI. Madrid: Santillana.

DURÁN, D.; TORRÓ, J. y VILA, J. (Coord.) (2003): Tutoría entre iguals. Un mètode d'aprenentatge cooperatiu per a la diversitat. Barcelona: ICE- UAB.

FORSTER, F; HOUNSELL, D. y THOMPSON, S (1994): Mentoring: a Handbook for Mentors in the Teaching Award Scheme. Edinburgh: Centre for Teaching, Learning and Assessment, in Association with the Faculty Group of Arts, Divinity and Music, University of Edinburgh.

FULLERTON, H (ed.) (1996): Facets of Mentoring in Higher Education 1. SEDA Paper, 94. Birmingham: SEDA Publications.

GAIRÍN, J.; QUINQUER, D.; FRANCH, J.; FEIXAS, M. y GUILLAMÓN, C. (2003): Un modelo para la generalización de un programa de tutoría universitaria. En Simposio de Estrategias de Formación para el cambio organizacional. Barcelona: Universitat Autònoma de Barcelona.

GAIRÍN, J.; QUINQUER, D.; FRANCH, J.; FEIXAS, M. y GUILLAMÓN, C. (2004): Acciones tutoriales para la Universidad. En III Symposium de Docencia Universitaria. Bilbao: Universidad de Deusto.

GALLEGO, S. (1997): Perfil del tutor universitario. En APODACA, P.; ARBIZU, F.; LOBATO, C. y OLALDE, C. (Comps.)(1997): Orientación universitaria y evaluación de la calidad. Universidad País Vasco.

GARCÍA BASALLOTE, M.; RODRÍGUEZ, G. y PAJARES, E. (1999): Un proyecto de mejora en la orientación universitaria. El programa tutor. Revista de Investigación Educativa, 17, 2.

HAGGAN, P.S. (2000): Transition counseling in the community college. Community College Journal, 24, 6, 427-442.

HERNÁNDEZ DE LA TORRE, M.E. (1998): La nueva acción tutorial en la enseñanza universitaria. Revista de Enseñanza Universitaria, 13, 79-88. Sevilla: ICE, Universidad de Sevilla.

KOWALSKY, R. y FRESKO, B. (2002): Peer tutoring for college students with disabilities. Higher Education Research y Development, 21, 3.

LOCKE, W. y GOODERSON, T. (1994): Student mentors in higher education helping to improve opportunities for school pupils. En Fullerton, H.: Facets of mentoring in higher education 1. SEDA. UK

QUINQUER, D. y SALA, C. (2002): L'atenció tutorial a l'Autònoma: situacions i propostes. Publicacions ICE-UAB.

SALMERÓN, H. y LÓPEZ PALOMO, V.L. (Coords.)(2000): Orientación educativa en las universidades. Grupo Editorial Universitario.

SÁNCHEZ, M.F. (1999): La orientación universitaria, veinticinco años después. Revista Española de Orientación y Psicopedagogía, 9 (15), 87-107.

SAUNDERS, D. (1992): Peer tutoring in higher education. Studies in Higher Education, 17 (2), 211-218.

Tutoría Universitaria. http://www.ub.edu/tutorsub/manual/index.htm. 